\section{The dependence of cyclofusion on orientation}

\author{
ROBERT P. O'SHEA and BORIS CRASSINI \\ University of Queensland, St. Lucia, Queensland, Australia
}

With his invention of the stereoscope in 1833, Wheatstone showed dramatically that singleness of vision does not necessarily involve stimulation of corresponding retinal points in the two eyes (e.g., Wheatstone, 1852). He found that when each eye is presented simultaneously with contours rotated by small but opposite amounts from vertical, a single contour is seen. This single contour has an orientation that is between those of the two monocular displays (allelotropia, Nelson, 1975) and is, moreover, seen as tilted in depth (see Figure 1a). There is some debate as to the manner in which such binocular cyclofusion occurs. Some authors (e.g., Ogle, 1950; Véronneau-Troutman, 1972) propose that the two eyes rotate about their respective lines of sight (cycloverge, according to the terminology of Burian \& von Noorden, 1974) in response to the dichoptic display. The result of these cyclovergent eye movements is to cause the dichoptic retinal stimuli to fall on corresponding retinal areas. The impression of depth must then arise from some other mechanism (e.g., afference from the extraocular muscles mediating the cyclovergence). Others (e.g., Kertesz, 1971, 1972, 1973a, 1973b; Nelson, 1975) assert that binocular fusion is neural in nature. Specifically, Barlow, Blakemore, and Pettigrew (1967) argue that although retinal areas may be geometrically noncorresponding, they are neurally corresponding in that the areas form the separate monocular receptive fields of the same binocular cortical neurons. Such disparity detectors are optimally activated by simultaneous stimulation of the noncorresponding monocular receptive fields, and may form the neural substrate of stereopsis. Analysis of the scatter of such receptive fields indicates that binocular cortical neurons in the cat accept much larger horizontal than vertical disparities (Barlow et al., 1967). In this note, we provide a demonstration that shows differences in the ability of the human visual system to accept horizontal and vertical disparities, and supports the fusion rather than the cyclovergence explanation.

The stereogram shown in Figure $1 \mathrm{~b}$ is a cross rotated by equal ( $4 \mathrm{deg}$ ) but opposite amounts in the left and right eye fields. If cyclovergence took place,

We thank Rod Ashton, Jack Broerse, and Bob Post for helpful comments on an earlier draft of this note. The authors' complete mailing address is: Department of Psychology, University of Queensland, St. Lucia, Queensland 4067, Australia.
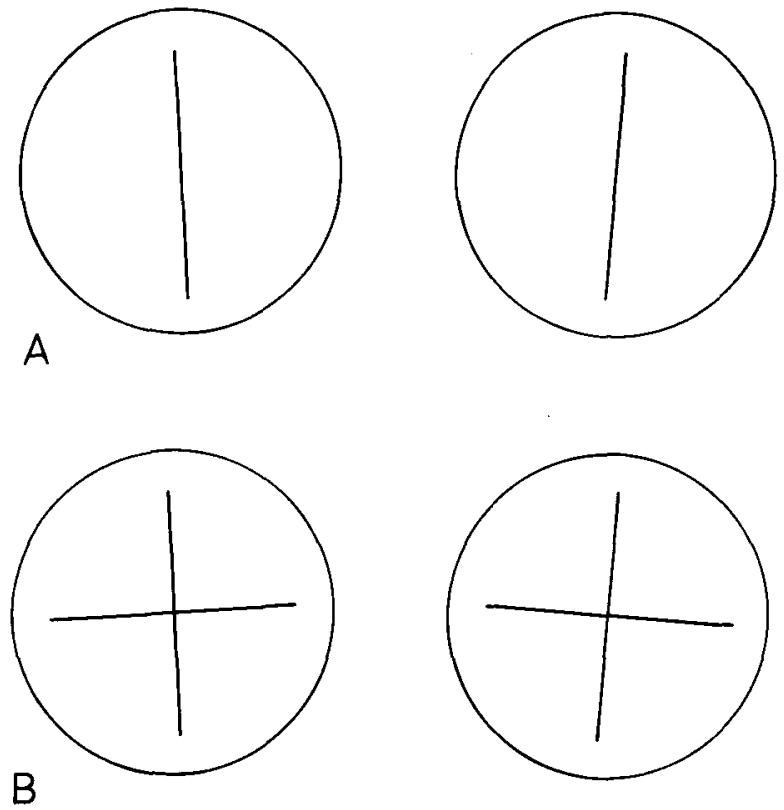

Figure 1. It is best to use a stereoscope to examine these two stereograms. There is some evidence that fusion by overconvergence may force a cyclovergence (Krekling, 1972), although the described effects are still clearly evident when fusion through overconvergence is employed. (a) This stereogram has been adapted from Wheatstone (1852). Each half image contains a single line rotated about its center by $4 \mathrm{deg}$ from vertical; the directions of rotation are opposite for the two eyes. Stereoscopic presentation of this figure should reveal a single line that is close to vertical and tilted in depth. (b) When this stereogram is fused, a single vertical contour should be seen as for Figure 1a. However, the nearhorizontal elements (each $4 \mathrm{deg}$ from horizontal) do not fuse and may even engage in binocular rivalry.

then the near-vertical and near-horizontal retinalimage components of the cross should fall on the (cyclorotated) vertical and horizontal meridians of each retina. The perceptual outcome of this would be a fused cross tilted in the frontoparallel plane. On the other hand, if fusion were to occur, then it would be expected that the vertical arms of the crosses would fuse (because the horizontal disparity associated with these components is within the limits of fusion; see O'Shea, Note 1). The horizontal arms would not fuse, because the vertical disparity associated with these components would not allow fusion (O'Shea, Note 1 ).

If Figure $\mathbf{l b}$ is observed stereoscopically, the resultant percept consists of a single vertical component, tilted in depth toward the observer, and two unfused near-horizontal components that may even demonstrate binocular rivalry. We have presented this display to a large number of observers (both naive and experienced in viewing stereoscopic material), all of whom reported this latter mixture of fusion and non- 
fusion. The above demonstration supports the notion that binocular fusion is neural in nature. ${ }^{1}$ Of course, it does not disallow a small amount of cyclovergence (Crone, 1971; Sullivan \& Kertesz, 1978); the crucial point is that cyclovergence must affect each arm of the cross equally. Therefore, the eye-movements hypothesis fails to explain the markedly different percepts for the horizontal and vertical components of the cross.

It has long been known that the fusional range for near-vertical contours is much greater than for nearhorizontal contours (Beasley \& Peckham, 1936). This has also been explained in terms of the ability of the two types of stimuli to evoke cyclovergence (Ogle, 1950). However, an alternative explanation is supported by physiological evidence of the larger horizontal than vertical disparity values that can be accepted by binocular cells (Barlow et al., 1967). The simple demonstration in Figure $1 \mathrm{~b}$ is consistent with these physiological data. The vertical arms of the dichoptic crosses are within the putative disparity tuning range of binocular cells, and are therefore signaled by these cells. The horizontal arms are unable to be processed by neurons sensitive to disparity and, indeed, produce binocular rivalry.

\section{REFERENCE NOTE}

1. O'Shea, R. P. Spatial determinants of binocular rivalry investigated with sine-wave gratings. Paper presented to the Experimental Psychology Conference, Monash University, Clayton, Victoria, Australia, June, 1974.

\section{REFERENCES}

Barlow, H. B., Blakemore, C., \& Petrigrew, J. D. The neural mechanism of binocular depth discrimination. Journal of Physiology, 1967, 193, 327-342.
Beasley, W. C., \& Peckhan, R. H. An objective study of "cyclotorsion." Psychological Bulletin, 1936, 33, 741-742.

Burian, H. M., \& von Noorden, G. K. Binocular vision and ocular motility. St. Louis: Mosby, 1974.

Crone, R. A. Human cyclofusional response. Vision Research, $1971,11,1357-1358$.

KaUfman, L. Sight and mind: An introduction to visual perception. New York: Oxford University Press, 1974.

KERTESz, A. E. More on human cyclofusional response: A reply to Crone's comments. Vision Research, 1971, 11, 1359.

KERTESz, A. E. Effect of stimulus complexity on human cyclofusional response. Vision Research, 1972, 12, 699-704.

Kentesz, A. E. Central components of fusional response: Effect of stimulus parameters. Vision Research, 1973, 13, 495-499. (a)

Kentesz, A. E. Disparity detection within Panum's fusional areas. Vision Research, 1973, 13, 1537-1543. (b)

KREKLING, S. Variation of stereoscopic acuity with observation distance and eye-movement characteristics. Perception \& Psychophysics, 1972, 12, 461-465.

NeLson, J. I. Globality and stereoscopic fusion in binocular vision. Journal of Theoretical Biology, 1975, 49, 1-88.

OGLE, K. N. Researches in binocular vision. Philadelphia: W. B. Saunders Co., 1950.

Sullivan, M. J., \& Kertesz, A. E. Binocular coordination of torsional eye movements in cyclofusional response. Vision Research, 1978, 18, 943-949.

Véronneau-Troutman, S. Cyclotropia. American Orthoptic Journal, 1972, 22, 36-43.

Wheatstone, C. Contributions to the physiology of visionPart the first. On some remarkable, and hitherto unobserved, phaenomena of binocular vision. Philosophical Magazine, $1852,3,241-267$.

\section{NOTE}

1. There is another explanation for the percept in terms of suppression theory as developed by Kaufman (1974). It is possible that there was selective suppression of the vertical arms but not of the horizontal arms. However suppression theory is unable to explain the depth in the display or the allelotropia (despite the invocation of fixation disparity to explain allelotropia in stimuli containing only one value of horizontal disparity).

(Manuscript received April 6, 1982; accepted for publication April 19, 1982.) 